formation with double sides; these are the treo original gcrm-layers. The space enclosed by the inner germlayer is the intestinal cavity; the whole formation we call gastrula after Haeckel. As the causes of the formation of the two germ-layers are the same for all animals consisting of more than one cell (matazoa), according to Götte's view, the form of development of the gastrula is therefore common to all, however indiscernible it may often be in the outside appearance. The cause of this is partly that the above-mentioned difference between the upper and lower hemispheres of the ovum varies in magnitude. If this difference is small, the result will be that only a moderate part of the lower hemisphere will be pressed inward, the inner germ-layer remaining simple, as for instance with the lower polypi, which on the whole consist of two layers of cells. As the energy of the inward pressure increases, a third germ-layer, the so-called middle one, is split off the stronger inner one; this third one, from being a simple intermediary layer, may develop and originate many and important organs. If in the dividing ovim only the difference referred to in the vertical axis exist, the gastrula is naturally formed equally in all directions between the two poles, so that if further transformations take place, these likewise occur equally in all directions from the intestinal cavity and its principal axis, and therefore in radiated planes or lines. Thus the difference in the first axis of the ovum, if it acts by itself, always leads to a radiated structure of body which we find with Polypi, Medusæ, Echinoidea, and their relatives. Yet the higher developed representatives of these classes already show here and there, and in unimportant points, indications of a transition to a higher type. If we suppose the two horizontal axes of the ovum to be unequal, then the formation of the gastrula must naturally be unequal likewise. The inequality, which with many of the Vermes already shows itself during the first divisions of the ovum, causes the gastrula to extend in one direction more than in any other, and thus to receive another principal axis. If at the same time the two sides precede in development the other parts, two symmetrical masses are formed, situated opposite one another (germ-streaks), and which approach each other more or less on the stomach side, and there produce certain principal organs. To this transverse divisions may be added, as in the Arthropoda; or this may not occur, as in the Viollusca. Vertebrata finally do not show the preponderance of the first formation on two opposite symmetrical sides of the ovum, but only on one, where the odd germ-streak is situated and indicates the future back. In a manner similar to that of the typical foundation of the embryo, Götte tries to deduce all other phenomena of development not from hypothetical causes of inberitance, but directly from the laws of the formation of the ovum; as, for instance, the whole development of the different organs and tissues. Any essential change in a ceriain animal species must then be deduced from a change in the laws of formation, which are peculiar to the ovum, i.e. its first cause lies in the ovum, and the live animal can never transfer newlygained changes of form directly to the law of formation of its germs, nor thus cause its descendants to inherit them.

\section{NEW DISCOVERY IN CONNECTION WITH THE POTATO DISEASE}

THERE has been hitherto one "missing link" in our 1 knowledge of the life-history of the potato-blight, Peronospora infestans. The non-sexual mode of reproduction by coniclia or zoospores has long beer known; but the sexual mode of reproduction has eluded observation. This link has now been supplied through the researches of Mr. Worthington Smith, who described his discovery in a paper read at the last meeting of the Scientific Committee of the Royal Horticultural Society, and published at length in the Gardener's Chronicle for July 1o. He finds the female organs, the "restingspores " or unfertilised "oospores," and the male organs or "antheridia," in the interior of the tissue of the tuber, stem, and leaf, when in a very advanced stage of decay; and he has actually observed the contact between the two organs in which the process of fecundation consists. In some remarks made at the meeting of the British Association last year by one of our high authorities, it was suggested that we have in the Peronospor $a$ an instance of the phenomenon not infrequent among fungi, known as "alternation of generations " and that the germination of the true spores of the potato-blight must be looked for on some other plant than the potato. Mr. Worthington Smith has, however, looked nearer home, and has proved that the suggestion is not at all events verified in all cases. It is matter of congratulation that, after the lapse of a period of nearly thirty years since the publication of the first important memoir on the subject, this discovery-important alike from a scientific and a practical point of view - has fallen to one of our own countrymen, notwithstanding the foreign aid invoked by the Royal Agricultural Society in settling the still unsolved problems connected with this perplexing pest.

\section{HISTORY OF THE PLAGIOGRAPH}

I SHOULD like to add a few words to my description of the instrument called the Plagiograph * (the $g$ to be pronounced soft, like $j$, as in Genesis, Plagiarist, Oxygen) in NATURE, vol. xii. p. I68, for the purpose of explaining the order of ideas in which it took its rise, and also a very beautiful extension of another recent kinematical invention to which it naturally leads the way, and which, thus generalised, I propose to term the Quadruplane.

The true view of the theory of linkagest is to consider every link as carrying with it an indefinitely extended. plane, and to look upon the question as one of relative

* It may be questioned whether a new-born child can have a history. Perhaps it might have been more correct to have used for my title, "History of the Birth of the Plngirgraph," but this would have been long; moreover, the Plagiograph proves to be an unusually precocious child, having in its very cradle given birth to a greater than itself, the Quadruplane, a fullgrown invention described in the sequel of the text.

It is quite conceivable that the whole universe may constitute one great linkage, i.e. a system of points hound to maintain invariable distances, certain of them from certain others, and that the law of gravitation and similar physical rules for reading off natural phenomena may be the consequences of this condition of things. If the Cosmic linkage is of the kind 1 have called complete, then deterninism is the law of Nature. but, if there be more than one degree of liberty in the system, there will be room reserver for the play of free-will. We shoud thus revert to the Aristotelian view uncler a somewhat wider aspect of circular (the most perfect because the simplest form of motion) being the primary (however recondite) law of cosmical dynamics. Speaking of cosmical laws brings to my mind a reflection I have made upon the new chemical theory of atomicity. Suppose it should turn out that the doctrine of Valence should be confirmed by experience, and that the consequent logico-mathematical theory of colligation containing the necessary laws of consecution, or if cone pleases so to say of cause and effect should plant its foot and introduce a firm basis of predictive science into chemistry, how beautiful will be the a firm basis of this and the physical law of inertia! which really merely affirms the fact of each atom or noint of matter cartying about with it a lertain the fact denoting its communicative and inverse receptive with it a certain number, such case Valency, also a ffirming a te the exact analogue in chemistry to Inertia capacity for colligation, will and might properly assume the name of chemical inertia. differ as egregiously as Isomers in their capacity for forming multifarious attachments.

I I believe it is to Mr. Samuel Roberts that we are indebted for the idea of passing from mere copulated links to planes associated with the links, and for the observation that the order of the corresponding Graphs is not thereby augmented. The substitution of the more general idea of linkage for link-work, and of isolating completely the conception of relative in lieu of absolute motion, is due to the author of these lines. Take the case of a Quadruplane in which the four joints in their natural order of sequence form a contra-parallelogram. It is well known (and the fact has been applied to machinery under the name of "the parallelogram of Reulleux") that the relative motion of an opposite pair of planes may be represented by causing two curves to roll upon each other; but I add that this may be done simultaneously for both pairs of planes, giving rise to a beautiful and previously unthought of double motion of rolling (without slip) between two ellipses for one pair and two hyperbolas for the other pair of planes. This is an immeone pair and two hyperbolas for the other pair of planes. This
diate deduction from the conception of purely relative motion.

Nотв-In the description of the plagiograph, for pointed parallelogram, p. 168, second column, line 14, lege jointed parallelogram. Also a dotted line should be drawn in Fig. $x$ connecting the points 0 and $\mathrm{P}^{\prime}$. 